IP Periodica Polytechnica

Mechanical Engineering

60(4), pp. 256-261, 2016

DOI: $10.3311 /$ PPme. 9688

Creative Commons Attribution (i)

RESEARCH ARTICLE

\section{Simulation of Natural Convection Heat Transfer of Nitrogen in a Cylindrical Enclosure}

\author{
Souheila Boutebba ${ }^{1 *}$, Wahiba Kaabar ${ }^{1}$
}

Received 06 July 2015; accepted after revision 05 July 2016

\begin{abstract}
In this work we consider the numerical simulation of heat transfer by natural convection in a horizontal cylindrical cell, filled with nitrogen gas and heated by a tungsten filament situated on its axis. The gas temperature range is from $400 \mathrm{~K}$ to the envelope of the lamp unto the $3000 \mathrm{~K}$ near tungsten wire. These temperatures create large temperature gradients (up to $500 \mathrm{~K} / \mathrm{mm}$ ) which produce complex patterns of currents circulating inside the cell. The physical principles governing this problem are modeled by the Navier-Stokes equations. These equations are solved numerically using the finite volume method. Numerical results show that the temperature gradients cause the generation of the thermal buoyancy force which induces a natural convection movement within the cell. The numerical results are in good agreement with those of an experimental study conducted by the Coherent Anti-Stokes Raman Scattering (CARS) technique with the same geometric, dynamic and thermal parameters.
\end{abstract}

\section{Keywords}

heat transfer, fluid flow, natural convection, computational fluid dynamics

\footnotetext{
${ }^{1}$ Department of Chemistry

Constantine 1 University

Route Ain El Bey 25017, Constantine, Algeria

${ }^{*}$ Corresponding author, email: bouteb.souha@gmail.com
}

\section{Introduction}

The problem of natural convection heat transfer over cylindrical or spherical enclosures is very frequent in several technological applications, such as vaporization and condensation of fuel droplets, nuclear reactor design, spray drying, solar energy collectors, and lamp technologies [1-11].

Several researchers have studied numerically and experimentally the natural convection heat transfer in spherical lamps, only limited literature is available for natural convection in cylindrical lamps [7, 12, 13]. Fisher and Fitzgerald [12] solved the conservation equations in two dimensions and predicted the flow patterns and tungsten transport in horizontal cylindrical lamps. The calculated temperature profiles showed good agreement with those obtained from a double exposure holographic technique. It was also demonstrated that lower gas filling pressures reduced the magnitude of convective effects.

Correa [13] calculated the movement of a fluid and heat transport in a tungsten halogen lamp using a curvilinear grid. This model was applied to vertically and horizontally oriented lamps and including an inert gas at high pressure. Makai et al. [14] studied a halogen lamp with a tungsten filament. The simulation of the free convection inside the lamp was carried by the energy equation and the Navier-Stokes equations. Based on the substantial partial differential equations of the free convection problem, this computer model was developed to evaluate the operating conditions in tungsten halogen lamps. The free convection was simulated by the convection-conduction equation and the incompressible Navier-Stokes equation. They determined velocity field, the temperature distribution and the pressure in the lamp. The pressure dependence on dimensionless characteristic numbers was evaluated. Different least square estimation methods for determination of distribution temperature and their applicability with regard to uncertainties of the spectral irradiance data of incandescent lamps were discussed by Rosenkranz et al. [15]. Recently Singh [11] studied numerically the natural convection heat transfer from a finned sphere in laminar and turbulent flow regimes using CFD (Fluent 6.3).

The aim of the present study is to simulate heat transfer by natural convection in a horizontal cylindrical cell filled with 
nitrogen gas and heated by a tungsten filament located centrally in it. Two models were considered for this study, a two-dimensional model (2D model) for a preliminary investigation and a three-dimensional (3D model) for a more general study. The effect of the gas filling pressure on the convective fluid flow is investigated. The temperature distribution within the optical cell is examined for various filament temperatures. To simulate end effects, the three dimensional model is considered. The experimental data provide 2D and 3D models validation. This work has been carried out using the CFD (Computational Fluid Dynamics) commercial code Fluent [16].

\section{Mathematical modelling}

In the present study, the fluid is regarded as Newtonian and incompressible; the flow is laminar in steady state. Radiation heat transfer is negligible. Under these assumptions the CFD model involves solving the continuity equation and momentum, which are expressed as:

Continuity equation

$$
\frac{\partial \rho}{\partial t}+\operatorname{div}(\rho u)=0
$$

Momentum conservation

- Momentum along X:

$$
\frac{\partial(\rho u)}{\partial t}+\operatorname{div}(\rho u \mathrm{u})=-\frac{\partial p}{\partial x}+\operatorname{div}(\mu \operatorname{gradu})+S_{M x}
$$

- Momentum along Y:

$$
\frac{\partial(\rho v)}{\partial t}+\operatorname{div}(\rho v u)=-\frac{\partial p}{\partial y}+\operatorname{div}(\mu \operatorname{grad} v)+S_{M y} .
$$

- Momentum along Z:

$$
\begin{aligned}
\frac{\partial(\rho w)}{\partial t}+\operatorname{div}(\rho w u) & =-\frac{\partial p}{\partial z}+\operatorname{div}(\mu \operatorname{grad} w)+S_{M z} . \\
S_{M x} & =-\rho g, S_{M y}=0 \text { and } S_{M z}=0 .
\end{aligned}
$$

The finite volume method is used for the numerical solution of these equations. The details of the application of the numerical method are documented in the work of Patankar [17].

Commercial CFD code Fluent $\left(\right.$ Fluent $^{\circledR}$ 6.3.26) is used to perform simulations. The governing equations are solved using pressure based two and three dimensional solver. Laminar viscous model is chosen for getting the flow field across the computational domain. Double precision calculations with second order upwind discretisation scheme are employed except for the pressure where STANDARD is selected. The SIMPLE algorithm is chosen for the pressure-velocity coupling. Convergence of numerical solution is ensured by monitoring the scaled residuals to a criterion of $10^{-5}$ for the continuity and momentum parameters.

\section{Problem definition}

The optical cell is assumed to be a long cylinder with an axial tungsten wire having a diameter of $1.58 \mathrm{~mm}$ and a length of $23.5 \mathrm{~mm}$, the wire is modeled as a solid cylinder. The cell envelope is defined to be $75 \mathrm{~mm}$ in diameter and $250 \mathrm{~mm}$ in length. The lamp's geometry is defined in three dimensions and molded in the horizontal orientation. The symmetry of the problem reduces the computational domain to the half of the total volume of the lamp. The boundary conditions are defined as follows:

Wall temperature $=400 \mathrm{~K}$

Filament temperature $=3000 \mathrm{~K}$

Fluid $=$ nitrogen gas

\subsection{Two-dimensional model: 2D Model}

We consider, firstly, a two-dimensional model of the lamp. A non-uniform circular grid of quadrilateral cells is constructed with 38000 nodal points, verified to give mesh independent results, where the largest number of meshes is concentrated in regions with large variations in temperature gradients, i.e zones near the filament (Fig. 1).

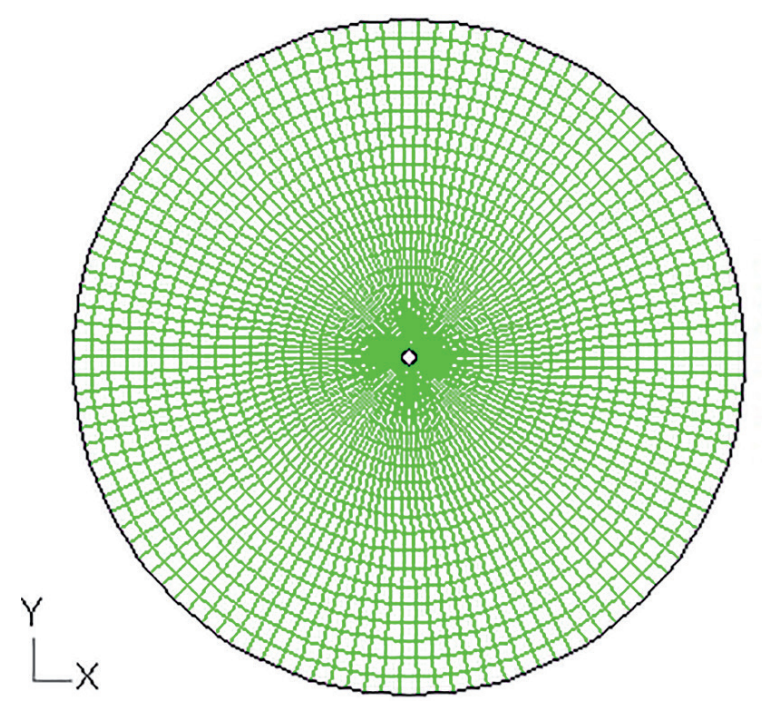

Fig. 1 Geometry and mesh (2D)

\subsection{Three-dimensional model: 3D Model}

The lamp geometry is defined by exploiting the symmetry plane perpendicular to the filament and passing through its center, the computational domain is reduced to $1 / 2$ of the total volume of the lamp (Fig. 2).

An axisymmetric plane is selected at the cross section of the lamp. For other boundaries we use the same boundary conditions as in the two-dimensional case.

A non-uniform quadrilateral meshes with a number of 333571 nodes is used (Fig. 2). A refinement of the mesh near the filament in the axial and radial direction is considered to take into account strong variations occurring in these areas. 


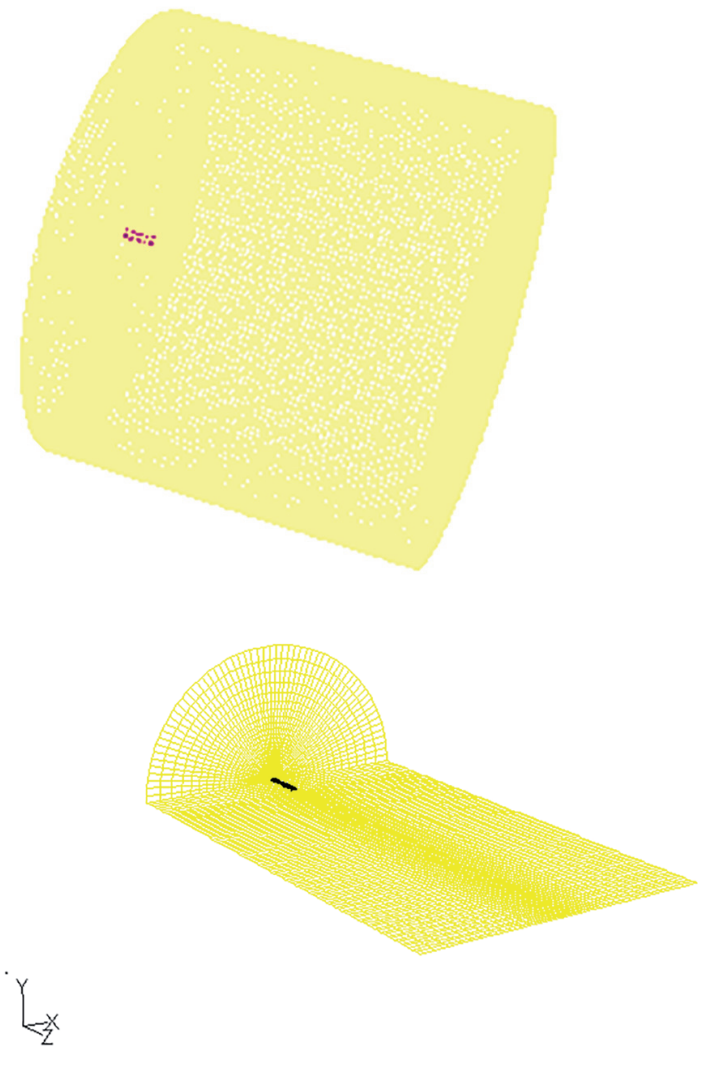

Fig. 2 Geometry and mesh (3D)

\section{Results}

\subsection{Tow-dimensional model}

The simulation was run until convergence after 700 iterations.

Pressure contours simulated (Fig. 3a) show that the minimum pressure is reached in the region above the filament where thermal buoyancy forces accelerate the gas to the top of the enclosure. Once reaching the wall where the pressure is maximal, the flow changes direction and follows a downward movement along and parallel to the side wall of the enclosure.

The thermal field is shown in Fig. 3b. Significant temperature gradients exist in the filament region (about $520 \mathrm{Kmm}^{-1}$ ), particularly, below the filament where the temperature field is slightly compressed by the current model. In Fig. 3b it is found that, radially away from the filament, the temperature decreases considerably especially near the wall. However, the temperature distribution is not axially symmetrical and this is due to the effect of convective fluid motion.

The velocity field exposes the model of free convection flow around a cylinder heated within an enclosure; the current shows the plume due to the thermal buoyancy. The relatively hotter fluid near the filament is entrained in an upward movement towards the upper of the envelope.

Once reaching the wall, the flow is divided into two parts which are deflected to the right and left of the vertical plane of symmetry, along and parallel to the lateral wall of the cell. At the bottom of the lamp, the two parts of the flow are together in upward movement parallel to the vertical plane of symmetry. The movement described is represented in Fig. 3c. The maximum gas velocity is equal to $0.243 \mathrm{~m} / \mathrm{s}$ and occurs at about $1 \mathrm{~cm}$ to the side of the filament, indicating significant convective currents occur near the filament. After passing through the enclosure, the cooled gas is mixed in the hot region of the filament; this causes a decrease in temperature values which involves currents less viscous and therefore higher velocities.

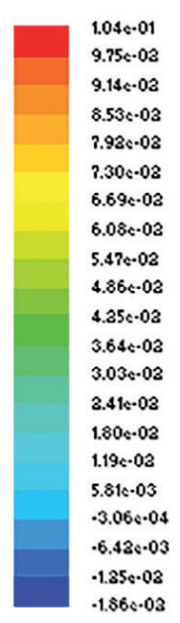

Pressure (Pa)

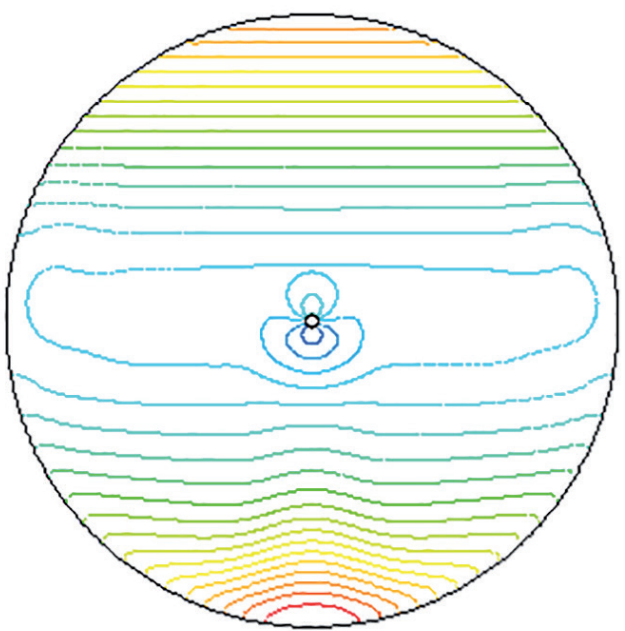

(a)

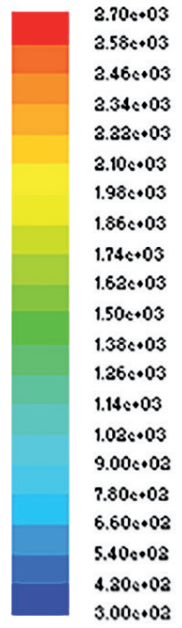

T emperature (K)

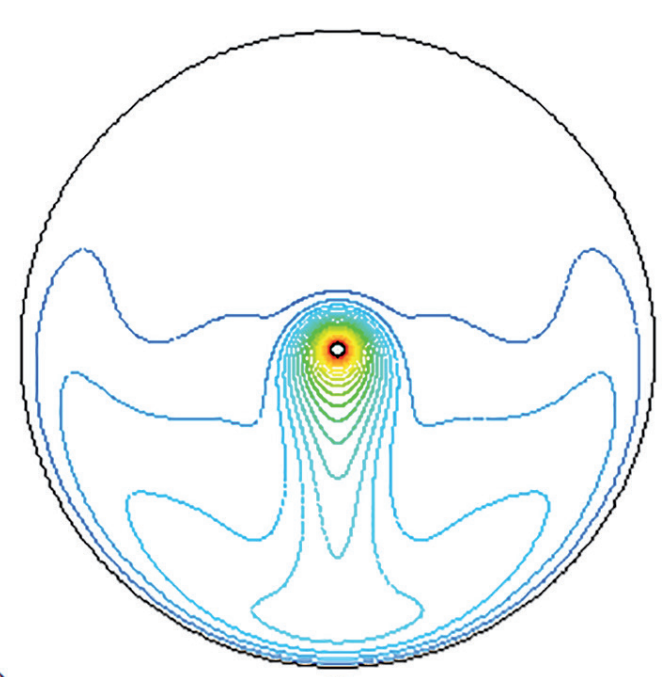

(b)

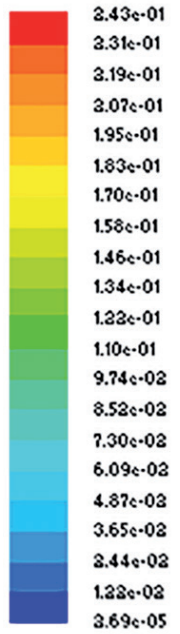

$\mathrm{V}$ elocity $(\mathrm{m} / \mathrm{s})$

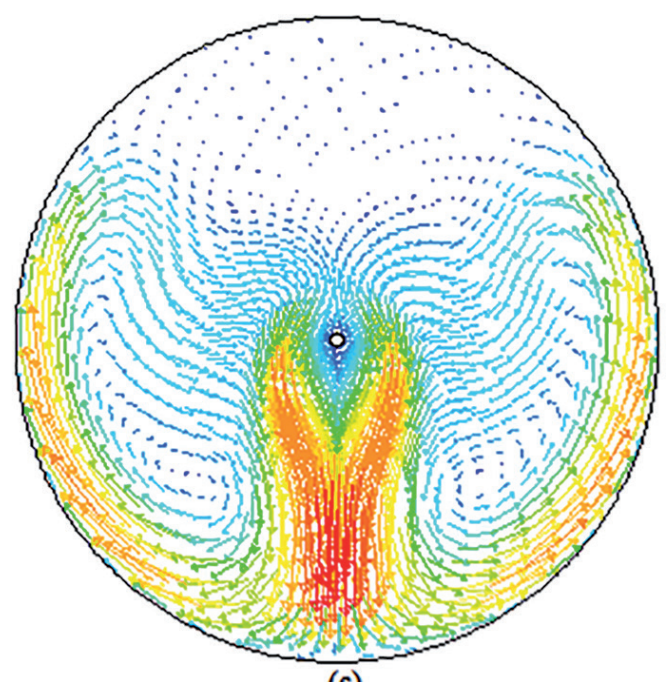

(c) 


\subsubsection{Effect of the pressure variation}

In this section we show the effect of varying the gas filling pressure within the enclosure by varying the operating pressure in fluent. The study is made for four different pressure values: $68.10^{2} \mathrm{~Pa}-25.10^{3} \mathrm{~Pa}-66.10^{3} \mathrm{~Pa}-10^{5} \mathrm{~Pa}$. sure are shown in Fig. 4. It can be seen that decreasing the pressure causes a gradual expansion of concentric contours of the filament region to the cell wall, until $\mathrm{P}=68.10^{2} \mathrm{~Pa}$ where only concentric temperature contours are observed throughout the volume of the enclosure. Reducing pressures below the atmosphere, leads to lowering the gas densities and the thermal buoyancy forces of the free convection. The effect of natural convection becomes smaller.

\subsubsection{Effect of the filament temperature variation}

In this section we show the gradual lowering effect of the filament temperature from $3000 \mathrm{~K}$ to the following temperatures: $2500 \mathrm{~K}, 2000 \mathrm{~K}, 1500 \mathrm{~K}, 1000 \mathrm{~K}, 500 \mathrm{~K}$. The filament is
The temperature contours for various values of the pres-

considered isotherm. It can be seen from Fig. 5 that moving away from the filament center in the vertical displacement (Y plane); the temperature decreases very rapidly. This rapid decrease may be caused by conduction, since the convection is a long process. The gas surrounding the filament gets hot and molecules constituting the gas acquire great kinetic energy. In this region; region of Langmuir, the heat transfer occurs through the collision by momentum exchange, rather than by convection. After this region we distinguish a temperature plateau caused by a balance between the conduction and convection. The presence of the plateau is maintained for various filament temperatures and becomes greater when the filament temperature decreases.

\subsection{Three-dimensional model}

Figure 6 shows the temperature contours in various cross sections mainly at the filament center $(z=0)$ and the filament end $(z=11.75 \mathrm{~mm})$.
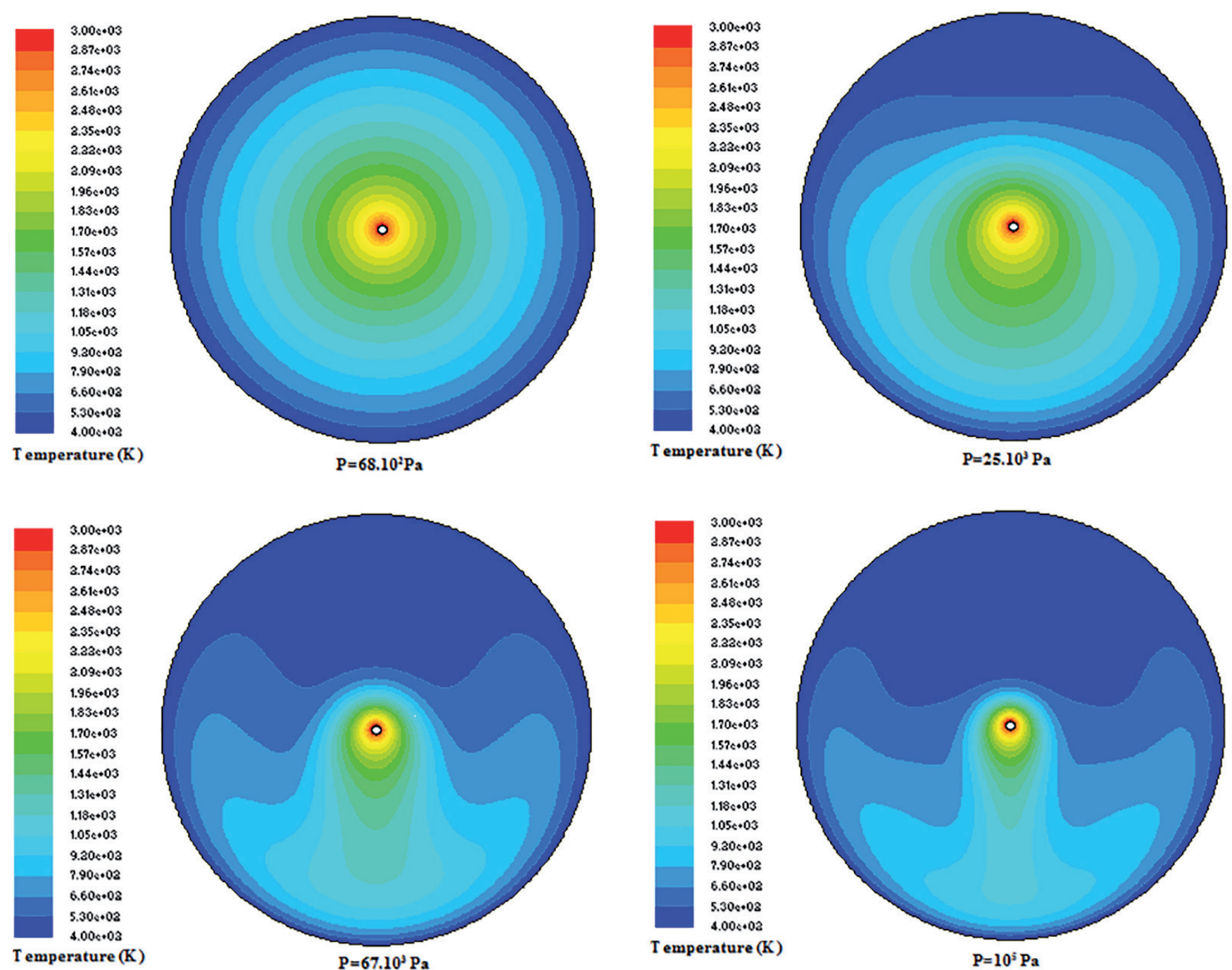

Fig. 4 Temperature contours for various values of pressure 


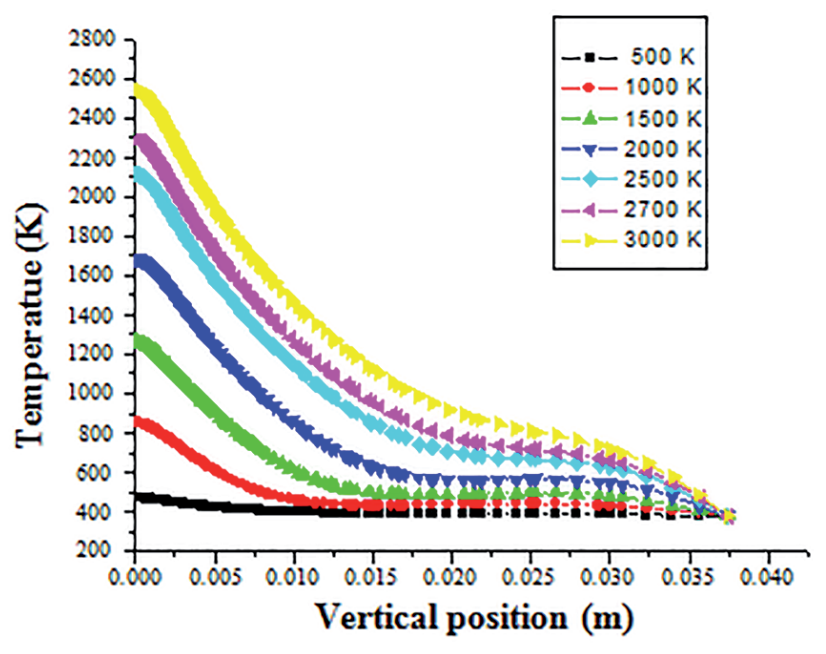

Fig. 5 Temperature variation in the vertical displacement for different filament temperatures
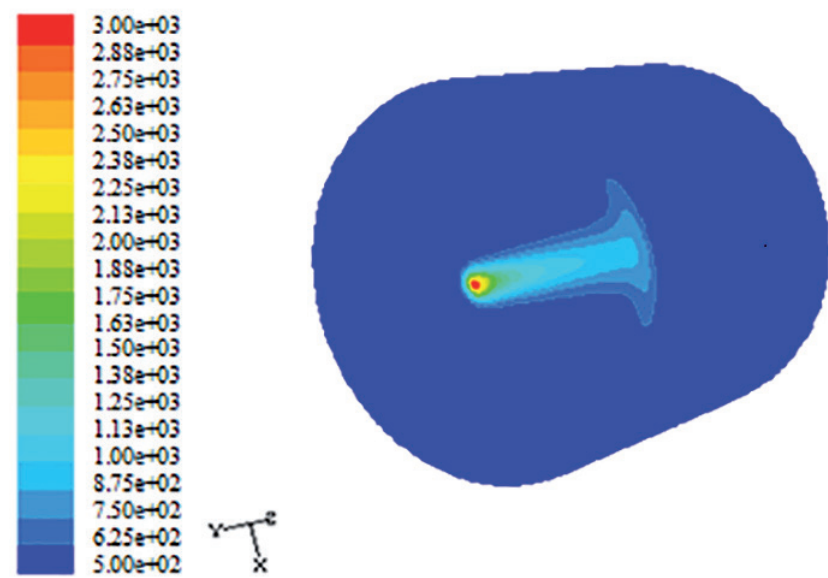

T empeature (K)

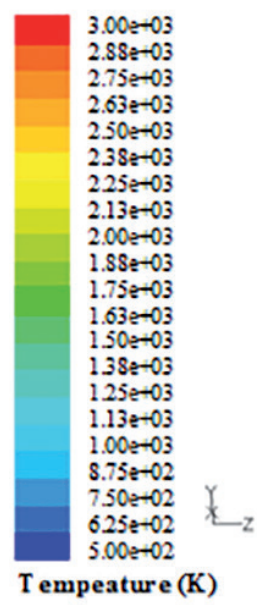

Fig. 6 Temperature contours

In the plane $\mathrm{z}=0 \mathrm{~mm}$, the convective effect is more significant than in the corresponding 2D case. Further, moving away from the filament center to the enclosure wall, the convection effects become less important until a nearly stagnant region of gas at the enclosure wall is reached.
Figure 7 shows that the highest current velocities occurring around the filament ends where the strongest temperature gradients are encountered. The velocity components cause a mixing of cooler gas from the lamp end into the hot filament region. This cooling effect is the lowering cause of the average gas temperatures predicted compared to that of the $2 \mathrm{D}$ case which results in a stronger convection effects. The maximum velocity is predicted to be in the plane perpendicular to the filament mid-position with magnitude equal to $0.406 \mathrm{~m} / \mathrm{s}$ as opposed to a value of $0.243 \mathrm{~m} / \mathrm{s}$ in the corresponding $2 \mathrm{D}$ case.
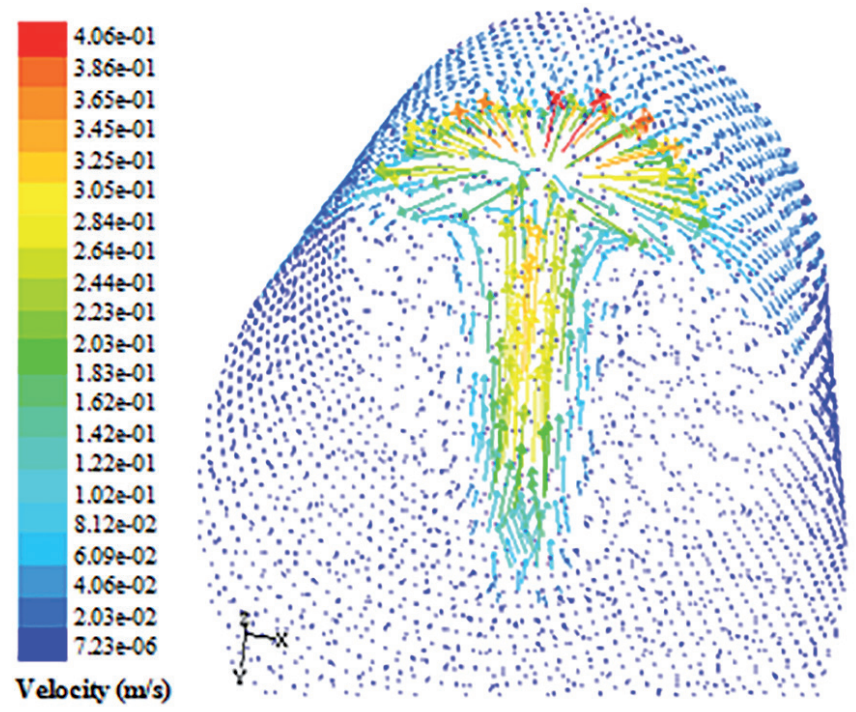

Fig. 7 Velocity vectors

The high thermal gradients that occur around the filament ends generate strong density gradients as shown in Fig. 8. This figure represents the gas density as a function of the horizontal displacement along the filament axis starting at the filament mid-point for different filament temperatures. It is found that the variation effect of the temperature filament on the density gradient is not important.

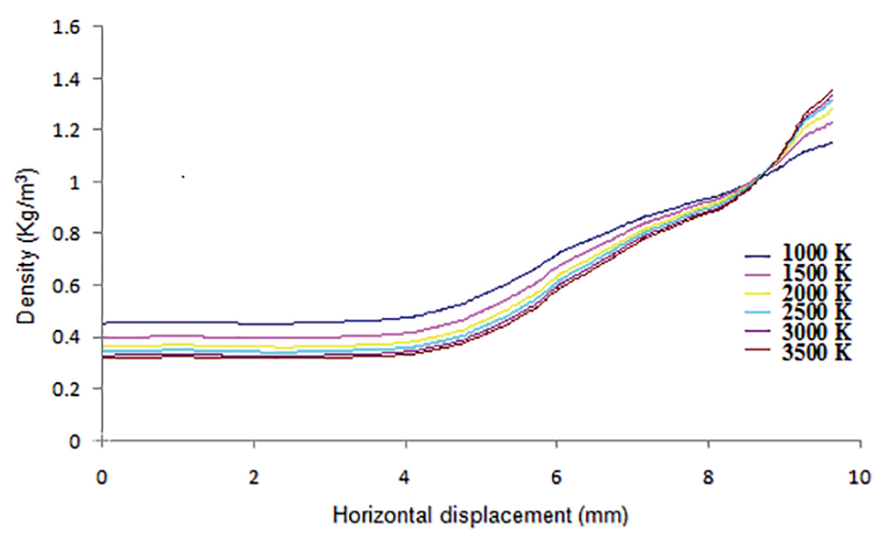

Fig. 8 Density variation with the horizontal displacement for different filament temperatures 


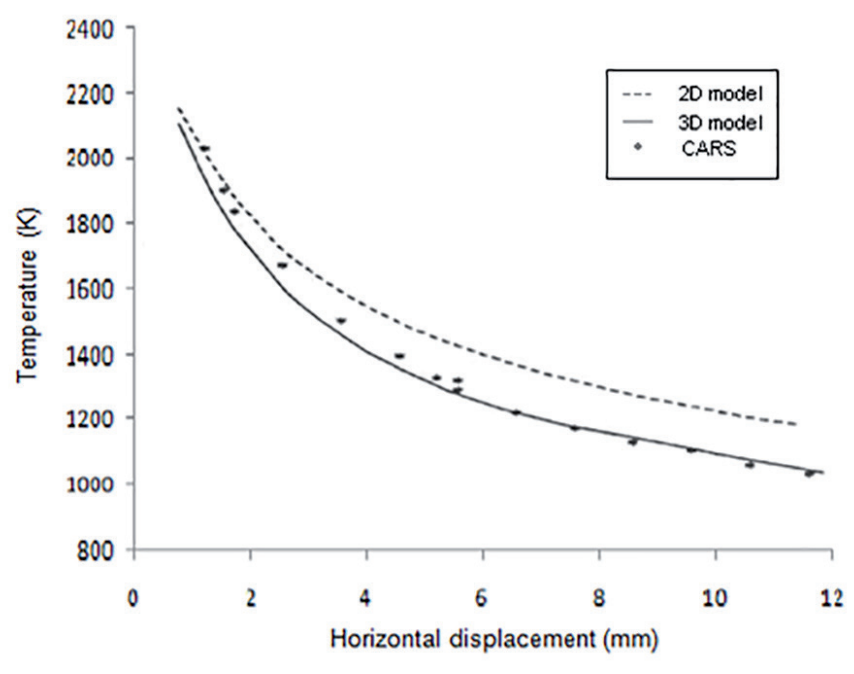

Fig. 9 Temperature comparison for 2D, 3D models and the experimental technique CARS

Figure 9 shows the temperature profiles above and along the filament in the horizontal cell, derived by the experimental technique CARS [18] and those predicted by the 2D and $3 \mathrm{D}$ models. The qualitative behavior of the temperature distribution is similar for both models with the experimental data. However, the 3D model, which accounts for end effects, is in good agreement with the experimental result.

\section{Conclusion}

This paper has described a modeling investigation of a two and a three-dimensional heat transfer and fluid flow inside a high temperature optical cell using CFD. The convective fluid flows are driven by the hot tungsten filament located centrally in the cylindrical cell which contains nitrogen gas.

The numerical results of this study show that the temperature gradients cause the generation of the thermal buoyancy force which induces a natural convection movement within the enclosure. It is found that reducing pressures below atmospheric amounts leads to decreasing the natural convection within the cell. The study addresses the influence of the filament temperature which shows that convection is more important for high filament temperature because of strong temperature gradients.

The results obtained by the $3 \mathrm{D}$ model demonstrate strong density gradients at the filament ends where the highest temperature gradients are found. The end effects lead to compressed structures in both temperature and velocity distributions.

The important outcome of the work is the observation of the end effects, due to the small length of the wire compared to the enclosure, on the temperature profile.

The study presented here has shown that the $2 \mathrm{D}$ investigation gives generally a good account of the magnitude and spatial distribution of the temperature. However, 3D simulation is more accurate.

\section{References}

[1] Haldar, S. C. "Laminar free convection around a horizontal cylinder with external longitudinal fins." Heat Transfer Engineering. 25(6), pp.45-53. 2004. DOI: 10.1080/01457630490486265

[2] Hadjadj, R., Kaabar, W. "Theoretical Modeling of Chemically Reacting Flows." International Journal of Scienc and Research. 3(6), pp. 2531-2535. 2014.

[3] Grosch, H., Fateev, A., Nielson, K. L., Clausen, S. "Hot gas flow cell for optical measurements on reactive gases." Journal of Quantitative Spectroscopy and Radiative Transfer. 130, pp. 392-399. 2013.

DOI: 10.1016/j.jqsrt.2013.06.029

[4] Sekhar, T. V. S., Hema Sundar Rajub, B., Murthy, P. V. S. N. "Higher order compact scheme for laminar natural convective heat transfer from a sphere." Applied Mathematical Modelling. 40(3), pp. 2039-2055. 2016. DOI: 10.1016/j.apm.2015.09.054

[5] Kim, T. H., Kim, D. K., Do, K. H. "Correlation for the fin Nusselt number of natural convective heat sinks with vertically oriented plate-fins." Heat Mass Transfer. 49(3), pp. 413-425. 2012. DOI: 10.1007/s00231-012-1100-0

[6] Yu, Z. T., Fan, L. W., Hu, Y. C., Cen, K. F. "Prandtl number dependence of laminar natural convection heat transfer in a horizontal cylindrical enclosure with an inner coaxial triangular cylinder." Heat and Mass Transfer. 53(7-8), pp. 1333-1340. 2010. DOI: 10.1016/j.ijheatmasstransfer.2009.12.027

[7] Es Sakhy, R., El Omari, K., Le Guer, Y., Blancher, S. "Rayleighe-Bénard -Marangoni convection in an open cylindrical container heated by a nonuniform flux." International Journal of Thermal Sciences. 86, pp. 198209. 2014. DOI: 10.1016/j.ijthermalsci.2014.06.036

[8] Logerais, P. O., Bouteville, A. "Modelling of an infrared halogen lamp in a rapid thermal system." International Journal of Thermal Sciences. 49(8), pp. 1437-1445. 2010. DOI: 10.1016/j.ijthermalsci.2010.03.003

[9] Zenebe, D. M, Matti, L. "Investigation on nature of waste heat from incandescent light bulbs." In: 10th International Conference on Environment and Electrical Engineering. Rome, Italy, May 8-11, 2011, pp. 1-4. DOI: 10.1109/eeeic.2011.5874653

[10] An, B. H., Kim, H. J., Kim, D. K. "Nusselt number correlation for natural convection from vertical cylinders with vertically oriented plate fins." Experimental Thermal Fluid Science. 41, pp. 59-66. 2012. DOI: 10.1016/j.expthermflusci.2012.03.010

[11] Singh, B., Dash, S. K. "Natural convection heat transfer from a finned sphere." International Journal of Heat and Mass Transfer. 81, pp. $305-$ 324. 2015. DOI: 10.1016/j.ijheatmasstransfer.2014.10.028

[12] Fisher, E., Fitzgerald, J. "Heat and mass transport in cylindrical gas-field incandescent lamps." Journal of Applied Physics. 45(7), pp. 2895-2902. 1974. DOI: 10.1063/1.1663697

[13] Correa, S. M. "Fluid flow and heat transfer in incandescent lamps." International Journal of Heat and Mass Transfer. 30(4), pp. 663-672. 1987. DOI: 10.1016/0017-9310(87)90196-7

[14] Makai, L., Hárs, G., Varga, G., Fülöp G., Deák, P. "Computer simulation of the operating pressure of tungsten halogen lamps." Journal of Physics D. Applied Physics. 38(17), pp. 3217-3226. 2005. DOI: 10.1088/0022-3727/38/17/S25

[15] Rosenkranz, P., Matus, M., Rastello, M. L. "On estimation of distribution temperature." Metrologia. 43(2), S130-S134. 2006.

DOI: $10.1088 / 0026-1394 / 43 / 2 /$ S26

[16] Fluent.Inc., Fluent documentation, www.fluent.com.

[17] Patankar, S. V. "Numerical Heat Transfer and Fluid Flow." McGrawHill, 1980. URL: http://www.ewp.rpi.edu/hartford/ ernesto/F2012/ CFD/Readings/Patankar-NHTFF-1980.pdf

[18] Devonshire, R., Dring, I. S., Hoey G., Porter, F. M., Williams D. R., Greenhalgh, D. A. "Accurate cars measurement and fluid-flow modeling of the temperature distribution around a linear incandescent filament." Chemical Physics Letters. 129(2), pp. 191-196. 1986. DOI: 10.1016/0009-2614(86)80195-6 